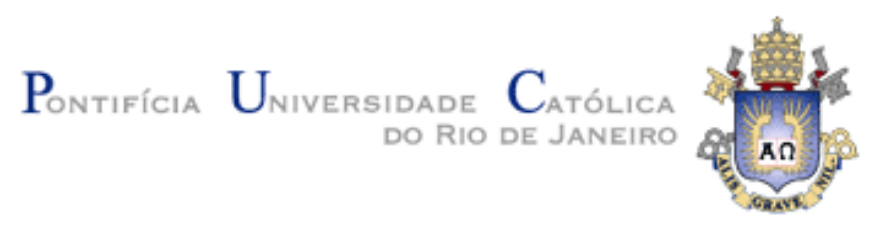

Aída Pereira Baêta

\title{
Caracterização da Matéria Orgânica Sedimentar no Sistema de Ressurgência de Cabo Frio
}

Tese de Doutorado

Tese apresentada como requisito parcial para obtenção do título de Doutor pelo Programa de PósGraduação em Química da PUC-Rio.

Orientadores: Prof ${ }^{a}$. Angela de Luca Rebello Wagener Prof. Renato da Silva Carreira 


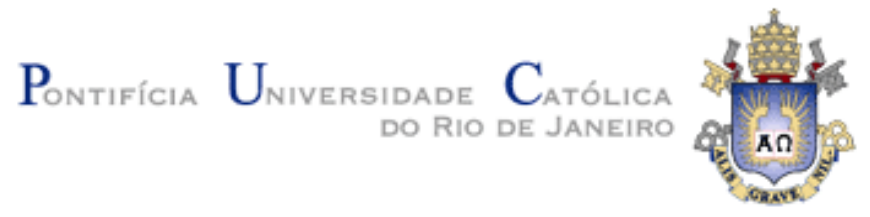

Aída Pereira Baêta

\section{Caracterização da Matéria Orgânica Sedimentar no Sistema de Ressurgência de Cabo Frio}

Tese apresentada como requisito parcial para obtenção do título de Doutor pelo Programa de Pós-Graduação em Química da PUC-Rio. Aprovada pela Comissão Examinadora abaixo assinada.

Profa. Angela de Luca Rebello Wagener Orientador Departamento de Química - PUC-Rio

Profa. Eliane Gonzalez Inst. de Estudos do Mar Alm. Paulo Moreira

Profa. Ana Luíza Spadano Albuquerque Departamento de Geoquímica - UFF

Dra. Cássia de Oliveira Farias Departamento de Química - PUC-Rio

Dro. Arthur de Lemos Scofield Departamento de Química - PUC-Rio José Eugenio Leal Coordenador(a) Setorial do Centro Técnico Científico - PUC-Rio Rio de Janeiro, 31 de julho de 2008 
Todos os direitos reservados. É proibida a reprodução total ou parcial do trabalho sem autorização da universidade, da autora e do orientador.

\section{Aída Pereira Baêta}

Graduou-se em Química Industrial na UFRuralRJ em 2001. Tornou-se Mestre em Química Analítica pelo Departamento de Química da PUC-Rio em 2004, mesmo ano em que iniciou seu curso de Doutorado em Química Analítica na mesma instituição. Participou de congressos nacionais e atualmente presta serviços de Consultoria pela PUC-Rio na Petrobrás.

Ficha Catalográfica

Baêta, Aída Pereira
Caracterização da matéria orgânica sedimentar no
sistema de ressurgência de Cabo Frio / Aída Pereira Baêta
; orientadora: Angela de Luca Rebello Wagener ; co-
orientador: Renato da Silva Carreira. - 2008.
322 f. : il. ; 30 cm
Tese (Doutorado em Química)-Pontifícia
Universidade Católica do Rio de Janeiro, Rio de Janeiro,
2008. $\quad$ Inclui bibliografia
1. Química - Teses. 2. Ressurgência. 3. Cabo
Briomarcadores moleculares. 5. N-Alcanos. 6.
Esteróis. 7. Álcoois graxos. 8. Ácidos graxos. I. Wagener,
Angela de Luca Rebello. II. Carreira, Renato da Silva. III.
Pontifícia Universidade Católica do Rio de Janeiro.
Departamento de Química. IV. Título.

CDD: 540 
A minha mãe por todo amor, carinho e dedicação ao longo de toda a minha vida. 


\section{Agradecimentos}

A Deus por estar sempre presente em minha vida.

A Professora Angela Wagener, pela orientação, confiança e apoio na execução do trabalho.

Ao Co-orientador Renato Carreira, por me receber em seu laboratório e me apresentar a novas técnicas analíticas e equipamentos até então não utilizadas diariamente por mim.

Ao Dr. Arthur Scofield, pelas inúmeras dúvidas sanadas, pela interpretação de muitos espectros e pela paciência.

A Cássia, pela amizade, pelas palavras de estímulos nas horas difíceis, pelas dicas na metodologia. Foi muito bom ter conhecido e convivido com alguém como você.

A toda a equipe do LABMAN: Francine (que me ajudou muito no cromatógrafo), Adriana (sempre disposta a ajudar), Priscila, Ricardo, Wellington, Rafael.

Ao CNPq, CAPES e a PUC-Rio, pelo suporte financeiro.

Ao Departamento de Química, em especial à secretária Fátima Almeida.

A Prof ${ }^{a} \operatorname{Dr}^{\mathrm{a}}$ Isabel Moreira e a $\operatorname{Prof}^{\mathrm{a}} \operatorname{Dr}^{\mathrm{a}}$ Helena Kehrig, minhas orientadoras no mestrado, aprendi muito com vocês.

A todos do Laboratório de Oceanografia Química da UERJ, pelo auxilio ao longo de todo este trabalho. Um obrigada especial: ao Luís, Luciana,Michelle, Thalita, Natália, Nafisa, Luís (Zé) em fim a todos.

Aos meus queridos estagiários Luíz e Nafisa, por ficarem até mais tarde me ajudando, pelos feriados, pelo apoio e trabalho árduo...e como!!!!

Aos amigos...todos....Cláudia, Olívia (amigas ao longo da vida), Luciana, Luciana, Eliane, Claudinha, Patrícia, Raquel. Obrigada pelo incentivo e carinho.

A toda a minha família, especialmente aos meus pais. Essa conquista é dedicada a você mãe e a você pai (em memória). 
Ao meu irmão e primo-irmão, cunhada, sobrinhos, primos, tios...pela torcida e apoio.

E a todos que colaboraram para execução deste trabalho. 


\section{Resumo}

Baêta, Aida Pereira; Wagener, Angela de Luca Rebello e Carreira, Renato da Silva. Caracterização da Matéria Orgânica Sedimentar no Sistema de Ressurgência de Cabo Frio. Rio de Janeiro, 2008. 322p. Tese de Doutorado - Departamento de Química, Pontifícia Universidade Católica do Rio de Janeiro.

Sistemas de ressurgência costeira têm grande interesse científico, pois por seqüestrarem nutrientes, controlam a biologia e a pesca em áreas oceânicas vastas. O ecossistema de Cabo Frio (RJ) representa a principal área de ressurgência costeira no Brasil e uma exceção no oceano global já que a maioria das zonas de ressurgência localiza-se em sistemas de correntes da borda Leste do Oceano Atlântico. Esta ressurgência sazonal e regida pelos padrões de vento da região causa intermitente, mas significativa resposta biológica na coluna de água de Cabo Frio, propiciando o aumento das comunidades de fito e zooplânctônicas. $\mathrm{O}$ enriquecimento das águas é responsável pela proliferação de microorganismos no sedimento. Este acoplamento dos processos físicos e biológicos controla a transferência de material planctônico para os sedimentos, visto que não há uma fonte relevante de material terrestre para a região. Para averiguação de alterações nos processos de transferência ao longo de parte do Holoceno, possivelmente ligados a mudanças climáticas, foram utilizados biomarcadores moleculares lipídicos em sedimentos coletados em outubro/2002 em 3 pontos entre a latitude $23^{\circ} 11^{\prime} \mathrm{S}$ e longitude $41^{\circ} 47^{\prime} \mathrm{W}$, sendo utilizados para este estudo 2 pontos denominados CF02-01A e CF02-02A. Além dos marcadores moleculares quantificados no presente estudo foram também utilizados os marcadores isotópicos e a composição elementar obtidos por outros autores para auxiliar na interpretação dos resultados. Os marcadores utilizados foram os hidrocarbonetos alifáticos, os esteróis, os álcoois graxos e os ácidos graxos. Os n-alcanos foram quantificados em CG-DIC e os esteróis, ácidos e álcoois graxos em CG-EM. O método empregado permitiu quantificar e identificar 31 compostos na fração de 
ácidos graxos e 67 na fração neutra. No testemunho CF02-01A foram obtidos as seguintes concentrações médias: 97,40 $\pm 99,15 \mu \mathrm{g}$ g-1 COT para os n-alcanos; 93,39 \pm 37,00 $\mu \mathrm{g}$ g-1 COT para os esteróis; 81,32 \pm 40,65 $\mu \mathrm{g}$ g-1 COT para os álcoois graxos e $129,70 \pm 132,41 \mu \mathrm{g}$ g-1 COT para os ácidos graxos. Para o testemunho CF02-02A foram verificadas as seguintes concentrações médias: $128,7 \pm 213,11 \mu \mathrm{g}$ g-1 COT para os n-alcanos; $68,25 \pm 30,02 \mu \mathrm{g}$ g-1 COT para os

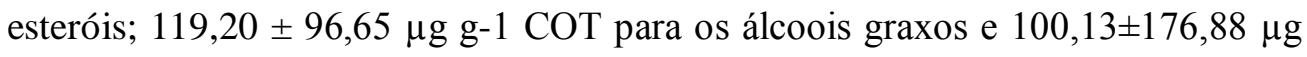
g-1COT para os ácidos graxos. Essas concentrações são inferiores às observadas nos sistemas de ressurgência africana ou da costa peruana. Os resultados obtidos demonstram que a matéria orgânica presente na região de Cabo Frio é fortemente influenciada por material autóctone altamente lábil derivado de diatomáceas e dinoflagelados, entretanto há também influência de matéria orgânica alóctone advinda, mais provavelmente, de transporte eólico. Observa-se um incremento na taxa de acumulação de marcadores autótones e alóctones no periodo entre cerca de 3600 BP e 2100 BP superposto a oscilações a intervalos de tempo da ordem de 300 anos. Tais variações devem estar associadas à variabilidade na circulação atmosférica regional ou semihemisférica, as quais podem controlar o fenômeno da

ressurgência. É importante ressaltar que foi observada neste estudo a presença de elevada MCNR, que é utilizada como indicativo da presença de resíduos de óleo degradado, o que foi confirmado pela presença de hopanos maturados na maioria das amostras. Isto demonstra a incidência de percolação de material oleoso a partir de reservatórios presentes na área.

\section{Palavras-chave}

Ressurgência, Cabo Frio, biomarcadores moleculares, n-alcanos, esteróis, álcoois graxos, ácidos graxos. 


\section{Abstract}

Baêta, Aida Pereira; Wagener, Angela de Luca Rebello e Carreira, Renato da Silva. Characterization of the sedimentary Organic Matter from Upwelling Sistem off Cabo Frio. Rio de Janeiro, 2008. 322p. Dr. Thesis Departamento de Química, Pontifícia Universidade Católica do Rio de Janeiro.

Upwelling coastal systems are of great interesting for science, since they provide nutrients and control the biology, the chemistry and fisheries in vast ocean areas. Cabo Frio ecosystem (RJ - located on the southwestern margin of the South Altantic Ocean) is the main coastal upwelling zone in Brazil and it is an exception in global ocean, as major upwelling zones are located in ocean current systems of East margins. This seasonal upwelling is governed by winds pattern prevailing in the area and it causes intermittent but significant biological responses in the water column of Cabo Frio. Besides generating rather intense phytoplankton and zooplankton surges the enriched waters are responsible for proliferation of microorganisms in the sediments. As long as there are no relevant sources of land derived nutrients to the region, the coupling of physical and biological ocean processes control the transfer of organic material of planktonic origin to the sediments. In this work, lipid biomarkers analyzed in sediments sampled in two stations (CF02-01 A and CF02-02A), located between $23^{\circ} 11^{\prime} \mathrm{S}$ and $41^{\circ} 47^{\prime} \mathrm{W}$ and collected in October/2002, were used to evaluate possible changes in the upwelling system during the late Holocene. Organic compounds were obtained through Soxhlet extraction technique, following saponification and the division in neutral and acid fractions. Neutral fraction was concentrated and then passed through a chromatographic column to separate n-alkanes from sterols, and fatty alcohols and acids. N-alkanes were quantified by GC-FID and sterols and fatty alcohols and acids by GC-MS. In fatty acid fraction 31 compounds were identified and quantified while in neutral fraction 67 compounds were investigated. CF02$01 \mathrm{~A}$ core was characterized by the average concentrations of $97,40 \pm 99,15 \mu \mathrm{g} \mathrm{g}^{-1}$ TOC for n-alkanes; 93,39 $\pm 37,00 \mu \mathrm{g} \mathrm{g}^{-1}$ TOC $^{-}$for sterols; $81,32 \pm 40,65 \mu \mathrm{g} \mathrm{g}^{-1} \mathrm{TOC}$ 
for fatty alcohols and $129,70 \pm 132,41 \mu \mathrm{g} \mathrm{g}^{-1}$ TOC for fatty acids. In CF-02A core the average concentrations obtained were: $128,7 \pm 213,11 \mu \mathrm{g} \mathrm{g}^{-1}$ TOC for n-alkanes; $68,25 \pm 30,02 \mu \mathrm{g} \mathrm{g}^{-1}$ TOC for sterols; $119,20 \pm 96,65 \mu \mathrm{g} \mathrm{g}^{-1}$ TOC for fatty alcohols and $100,13 \pm 176,88 \mu \mathrm{g} \mathrm{g}^{-1} \mathrm{TOC}^{-1}$ for fatty acids. These concentrations are smaller than the reported for upwelling systems in Africa and Peru. The results indicate that the organic matter in the Cabo Frio region is strongly influenced by highly labile autochthonous material derived from diatoms and dinoflagellates, although there is a significant influence of allochthonous organic matter possibly driven by eolic processes. An increase is observed in the accumulation rates of both autochthonous and allochthonous markers in the period between $3600 \mathrm{BP}$ and 2100 BP, superimposed by oscillations with time intervals of about 300 years. Such trends are probably bound to variability of regional or semi-hemispheric circulation of the atmosphere, which are controlling the upwelling processes. It is important to highlight the presence of substantive UCM in many sample, which allied to the occurrence of maturated hopanes indicate presence of degraded oil residues. This is a strong evidence of oil seepage from reservoirs abundantly present in the region.

\section{Keywords}

Upwelling, Cabo Frio, molecular biomarkers, n-alkanes, sterols, fatty alcohols, fatty acid. 


\section{Sumário}

1. Introdução 20

1.1. Introdução geral 20

2. Fundamentos Teóricos 23

2.1. A Importância da Matéria Orgânica 23

2.2. A Importância dos Marcadores Moleculares 26

2.2.1. n-alcanos 28

2.2.2. Esteróis 32

2.2.3. Álcoois Graxos $\quad 35$

2.2.4. Ácidos Graxos 36

3 Área de Estudo $\quad 40$

3.1. Localização da Área de Estudo 40

3.2. Características Climáticas 41

3.3. Características Oceanográficas 42

3.4. Caracterização da Matéria Orgânica no Sistema de

Ressurgência de Cabo Frio. 43

4. Metodologia 45

4.1. Amostragem e descrição dos testemunhos 45

4.2. Abertura dos Testemunhos e Descrição das Amostras 45

4.3. Geocronologia 47

4.4. Teores de Carbono Orgânico Total (COT), Nitrogênio Total e Determinação Isotópica de Carbono $\left(\delta{ }^{13} \mathrm{C}\right)$ e Nitrogênio $\left(\delta{ }^{15} \mathrm{~N}\right) \quad 48$

4.5. Determinação dos Marcadores Moleculares 48

4.5.1. Descontaminação do Material 48

4.5.2. Solventes e outros Materiais Utilizados neste Estudo 48

4.6. Testes Metodológicos 49

4.6.1. Teste de Metodologia para os Ácidos Graxos 49

4.6.2. Teste da Hidrólise Alcalina (Saponificação): Influência sobre a mistura de padrões autênticos de ácidos graxos - 37 Componentes

FAME Mix 
4.6.3. Teste de Metodologia para os Esteróis 52

4.6.4. Preparação da Amostra 53

4.6.5. Extração 53

4.6.6. Hidrólise Alcalina (Saponificação) 54

4.6.7. Fração Neutra (SAP-N) 54

4.6.8. Fração Ácida (SPA-A) 56

4.6.9. Determinação Quali-Quantitava para os n-Alcanos

Estudados $\quad 57$

4.6.10. Determinação Quali-Quantitativa para os Esteróis e

Álcoois graxos Estudados $\quad 62$

4.6.11. Determinação Quali-quantitativa para os Ácidos Graxos 74

5 Resultados e Discussão 79

5.1.1. Tratamento Estatístico dos Resultados 79

5.1.2. Composição Isotópica e Composição Elementar dos Perfis Sedimentares Estudados $\quad 79$

5.1.3. Classes Lipídicas Totais 82

5.1.4. Avaliação dos Resultados Obtidos para os n-alcanos. 83

5.1.5. Avaliação dos Resultados Obtidos para os Esteróis

Quantificados 96

5.1.6. Avaliação dos Resultados Obtidos para os Álcoois Graxos 116

5.1.7. Avaliação dos Resultados Obtidos para os Ácidos Graxos 124

5.1.8. Análise Fatorial (AF) 139

5.1.9. Análise do fluxo dos biomarcadores para os sedimentos amostrados

6. Conclusões 152

7. Referências Bibliográficas 156 


\section{Lista de Tabelas}

Tabela 1. Esteróis quantificados neste capítulo e suas respectivas fontes no ambiente marinho

Tabela 2: Relações entre a presença de um ácido graxo

e a informação geoquímica associada

Tabela 3. Código, número e profundidade das amostras coletadas para realização deste estudo

Tabela 4. Tabela contendo os padrões sub-rogados utilizados neste estudo para avaliação da recuperação analítica

Tabela 5. Condições Cromatográficas estabelecidas na determinação dos biomarcadores avaliados utilizando CG-DIC

Tabela 6. Tabela contendo os Limites de detecção e quantificação para a metodologia utilizada na determinação dos n-alcanos estudados

Tabela 7. Condições Cromatográficas estabelecidas na determinação dos biomarcadores (esteróis e álcoois graxos) avaliados utilizando CG-EM

Tabela 8. Tempos de retenção, íons de quantificação e íons de confirmação utilizados na identificação dos esteróis por CG-EM no sedimento de Cabo Frio

Tabela 9. Tabela contendo o limite de quantificação/detecçã dos esteróis individuais em aproximadamente $9,0 \mathrm{~g}$ peso seco de sedimento extraído para os testemunhos CF02-01A $(\mathrm{N}=57)$ e CF02-02A ( $\mathrm{N}=26)$

Tabela 10. Tempos de retenção, íons de quantificação e íons de confirmação utilizados na identificação dos álcoois graxos por CG-EM no sedimento de Cabo Frio

Tabela 11. Tabela contendo o limite de quantificação/detecção dos álcoois graxos $\left(\mathrm{C}_{18 \mathrm{OH}}\right.$ e $\left.\mathrm{C}_{19 \mathrm{OH}}\right)$ em aproximadamente 9,0 g peso seco de sedimento extraído para os testemunhos CF02-01A $(\mathrm{N}=58)$ e CF02-02A $(\mathrm{N}=29)$ 
Tabela 12. Condições instrumentais utilizadas no CG-DIC para a determinação dos ácidos graxos

Tabela 13. Tabela contendo os tempos de retenção, a nomenclatura usual e a nomenclatura IUPAC dos ácidos graxos quantificados neste estudo

Tabela 14. Análise estatística realizada entre os percentuais relativos dos ácidos graxos, álcoois graxos e n-alcanos de cadeia carbônica curta e longa a fim de verificar se esses compostos são provenientes de uma mesma fonte marinha ou terrestre. Teste de Spearman com $p<0,05$ e $95 \%$ de confiança

Tabela 15. Teste estatístico não paramétrico de correlação Spearman $(p<0,05)$ das idades calibradas em função do percentual relativo da MCNR, razão MCNR/Resolv, razão Ímpar/Par e índice IPC para os testemunhos CF02-01A ( $\mathrm{N}=49$ ) e CF02-02A ( $\mathrm{N}=28$ )

Tabela 16. Tabela contendo os resultados obtidos para os índices e as razões em função das idades calibradas (testemunho CF02-01A)

Tabela 17. Resultados da análise estatística de correlação Spearman dos $n$-alcanos, ao nível de significância $p<0,05$, para avaliar se há correlação significativa entre as razões e os índices determinados nos sedimento analisados. Os valores listados são os resultados obtidos para o $\mathrm{R}$ e são reportados apenas aqueles com confiabilidade $>95 \%$

Tabela 18. Tabela contendo os resultados obtidos para os índices e as razões dos n-alcanos em função das idades calibradas (testemunho CF02-01A)

Tabela 19. Correlação estatística de Spearman, com

probabilidade de erro $<5 \%(p<0,05)$, entre as razões de esteróis nos sedimentos estudados da região de Cabo Frio. Perfil CF02-01A ( $N=56)$. São reportados os valores de $R$ apenas para $p<0,05$ 
Tabela 20. Correlação estatística de Spearman entre as razões de esteróis nos sedimentos estudados da região de Cabo Frio. Perfil CF02-02A ( $\mathrm{N}=26$ ). São reportados os valores de $R$ apenas para $p<0,05$

Tabela 21. Razões e concentrações percentuais relativas obtidas entre os esteróis de origem terrestre (Campesterol/Estigmasterol/ßsitosterol) em função das idades calibradas no testemunho CF02-01A ( $N=56)$ coletado em Cabo Frio

Tabela 22. Razões e concentrações percentuais relativas para 0 campesterol, estigmasterol e $\beta$-sitosterol em função das idades calibradas no perfil CF02-02A $(N=26)$

Tabela 23. Resultados obtidos para o teste estatístico não-paramétrico de correlação Spearman $(p<0,05)$ entre os índices e as razões de álcoois graxos calculados para os testemunhos CF02-01A ( $\mathrm{N}=59)$ e CF02-02A ( $\mathrm{N}=29$ )

Tabela 24. Resultados obtidos para o teste estatístico de correlação Spearman $(p<0,05)$ entre os índices e as razões diagnósticas de ácidos graxos calculados para o testemunho CF02-01A ( $\mathrm{N}=35)$

Tabela 25. Resultados obtidos para o teste estatístico de correlação Spearman $(p<0,05)$ entre os índices e as razões diagnósticas de ácidos graxos calculados para 0 testemunho CF02-02A ( $\mathrm{N}=18)$

Tabela 26. Correlação obtida entre as variáveis agrupadas em seis fatores na análise fatorial entre os biomarcadores mais significativos no testemunho CF02-01A 


\section{Lista de Figuras}

Figura 1. Estrutura básica de esterol (Steraloids, 2006)

Figura 2. Visão geral Sul - Leste da plataforma continental brasileira (a) e no detalhe a região de ressurgência de Cabo Frio, incluindo os locais onde os perfis foram coletados (CF02-01A e CF02-02A) (b)

Figura 3. Abertura dos testemunhos CF02-01A e CF02-02A

Figura 4. Fluxograma da metodologia adotada para fração neutra do extrato

Figura 5. Exemplo de cromatograma obtido para a amostra M.sed 10 do perfil CF02-01A. No cromatograma estão também representados o padrão sub-rogado $n-\mathrm{dC}_{30}$ e o padrão interno de quantificação n- $\mathrm{dC}_{24}$

Figura 6. Exemplo de cromatograma demonstrando alguns dos esteróis (corrente total de íons - TIC) quantificados na amostra 01A_M.Sed.10 do perfil CF02-01A.

Psub - Androstanol; PI - Colestano; S5 - 24-nor-colesta-5,22-dien3 $\beta$-ol; S6 - 24-nor-colesta-22-dien-3 $\beta$-ol; S8 - Colest-5,22-

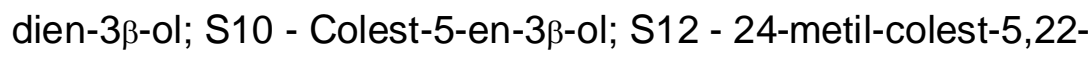

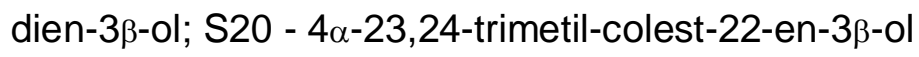

Figura 7. Estruturas químicas de alguns dos esteróis

identificados neste estudo. Fonte: Steraloids (2007)

Figura 8. Exemplo de cromatograma parcial com íons-chave para identificação e quantificação de álcoois graxos e fitol. Entre 15 e 41 min. A escala de abundância é ampliada em 10 vezes. $\mathrm{C}_{19 \mathrm{OH}}-$ Padrão sub-rogado;

$\mathrm{PI}$ - Colestano

Figura 9. Exemplo de cromatograma CG-FID dos ácidos graxos quantificados na amostra 01A - M.sed. 50 (3001 anos AP) do perfil CF02-01A 
Figura 10. Variação da razão $\mathrm{C} / \mathrm{N}, \delta{ }^{15} \mathrm{~N}$ e $\delta{ }^{13} \mathrm{C}$ em relação a idade ao longo do testemunho CF02-01A

Figura 11. Variação da razão $\mathrm{C} / \mathrm{N}, \delta{ }^{15} \mathrm{~N}$ e $\delta{ }^{13} \mathrm{C}$ em relação

a idade ao longo do testemunho CF02-02A

Figura 12. Perfis cromatográficos dos testemunhos sedimentares coletados em Cabo Frio. Em (a) Amostra 01A_M.sed 20 (1370 anos AP) e (b) Amostra 01A_M.sed 50 (3001 anos AP) perfil CF02-01A. Em (c) Amostra 02A_M.sed 14 (1659 anos AP) e (d) Amostra 02A_M.sed 35 (7243anos AP) perfil CF02-02A

Figura 13. Representação gráfica para os índices e razões em função das idades calendário para os testemunhos analisados. (a) Testemunho CF02-01A e

(b) CF02-02A

Figura 14. Perfil cromatográfico da amostra

01A_M.Sed 57 (3597 anos AP) do testemunho CF02-01A sedimentar coletado em Cabo Frio

Figura 15. Gráficos obtidos para razões e índices $\mathrm{x}$ idades calendário do perfil CF02-02A ( $\mathrm{N}=28$ ) amostrado em Cabo Frio (n-alcanos)

Figura 16. Gráficos obtidos para razões e índices x idades calendário do perfil CF02-01A ( $\mathrm{N}=49)$ amostrado em Cabo Frio (n-alcanos)

Figura 17. Gráficos contendo a concentração em percentual relativo do campesterol, estigmasterol e $\beta$-sitosterol em função das idades calibradas no testemunho CF02-01A ( $N=56)$ coletado na região de Cabo Frio

Figura 18. Gráficos demonstrando a variação das razões Colesterol/Fitosterol, Dinosterol/Metilenocolesterol e Colesterol/Metilenocolesterol em função das idades calendário para o testemunho CF02-01A ( $\mathrm{N}=56)$ 105

Figura 19. Gráficos demonstrando a variação das razões Campestanol/Campesterol, $\beta$-sitostanol/ $\beta$-sitosterol e 
Colestanol/Colesterol em relação às idades calendário

para o testemunho CF02-01A ( $\mathrm{N}=56)$

Figura 20. Gráficos contendo a concentração em percentual relativo do campesterol, estigmasterol e $\beta$-sitosterol em função das idades calibradas no testemunho CF02-02A ( $\mathrm{N}=26$ ) coletado na região de Cabo Frio

Figura 21. Gráficos demonstrando a variação das razões Colesterol/Fitosterol, Dinosterol/Metilenocolesterol e Colesterol/Metilenocolesterol em função das idades calendário para o testemunho CF02-02A (N 26)

Figura 22. Gráficos demonstrando a variação das razões Campestanol/Campesterol, $\beta$-sitostenol/ $\beta$-sitosterol e Colestanol/Colesterol em relação às idades calendário para o testemunho CF02-02A ( $\mathrm{N}=26$ )

Figura 23. Gráficos demonstrando a variação das razões e índices em relação às idades calendário ao longo do testemunho CF02-01 A ( $\mathrm{N}=59$ )

Figura 24. Gráficos demonstrando a variação das razões e índices em relação às idades calendário ao longo do testemunho CF02-02A ( $\mathrm{N}=29$ )

Figura 25. Gráficos demonstrando a variação das razões e índices de ácidos graxos em relação às idades calendário ao longo do testemunho CF02-01A ( $\mathrm{N}=56$ )

Figura 26. Gráficos demonstrando a variação das razões e índices de ácidos graxos em relação às idades calendário ao longo do testemunho CF02-02A $(N=12)$

Figura 27. Dendrograma dos biomarcadores individuais em função de suas informações geoquímicas no perfil CF02-01A

Figura 28. Representação das estações (idade calibrada do testemunho CF02-01A) nos planos fatoriais 1 e 2 da análise fatorial 
Figura 29. Representação das estações (idade calibrada do testemunho CF02-01A) nos planos fatoriais 1 e 4 da $A F$

Figura 30. Representação das estações (idade calibrada do testemunho CF02-01A) nos planos fatoriais 1 e 6 da AF

Figura 31. Representação das estações (idade calibrada do testemunho CF02-01A) nos planos fatoriais 2 e 4 da AF

Figura 32. Representação das estações (idade calibrada do testemunho CF02-01A) nos planos fatoriais 4 e 6 da AF

Figura 33. Gráficos demonstrando a variação no fluxo dos biomarcadores (terrestres e marinhos) e no fluxo de carbono orgânico em relação às idades calendário no testemunho CF02-01A 